\title{
Feature Level Two -Dimensional Arrays Based Fusion in the Personal Authentication system using Physiological Biometric traits
}

\author{
Jerusalin Carol .J ${ }^{1 *}$, A. Lenin Fred ${ }^{2}$, Ashy.V.Daniel ${ }^{2}$. \\ ${ }^{1}$ Research scholar, Department of Information and Communication Engineering, Anna University, \\ Chennai, India ${ }^{2}$ Department of CSE, Mar Ephraem College of Engineering and Technology, Marthandam, India.
}

\begin{abstract}
The fingerprint, knuckle print and the retina are used to authenticate a person accurately because of the permanence in the features. These three biometric traits are fused for better security. The fingerprint and knuckle print images are pre-processed by morphological techniques and the features are extracted from the normalized image using gabor filter. The retinal image is converted to gray image and pre-processing is done using top hat and bottom hat filtering. Blood vessels are segmented and the features are extracted by locating the optic disk as the centre point. The extracted features from the fingerprint, knuckle print and the retina are fused together as one template and stored in the data base for authentication purpose, thus reducing the space and time complexity.
\end{abstract}

Key words: Fingerprint, Knuckle print, Retina, Blood vessel segmentation, Feature level Fusion, Template.

\footnotetext{
*Author for correspondence: caroloct2@gmail.com
} 


\section{INTRODUCTION}

Biometrics is the system which can authenticate a person using their biological measure, for authentication and security purpose by identifying the query features with the predefined features. The retina, fingerprint and knuckle print are used due to their universality, uniqueness, permanence and high accuracy in their performance [1].The features of the fingerprint are classified as level1, level2 and level 3 features. The orientation and singular points are the level 1 features, ridge endings, bifurcation, crossover point, trifurcation are the features of level 2, pores, ridge contour, dots and island are the level 3 features. The scooping can be done with the level 1 and level 3 features, can only be extracted from high quality images. Due to these disadvantages, level 2 features are considered.

Fusion techniques are used for match score level fusion. In [2], the Measurement level or confidence level the scores are generated. Normalization is required to combine the scores and converted into common domain or scale called piecewise linear normalization. The piecewise linear normalization techniques are achieved by the EER of $2.79 \%$ and TER of 5.15\%.The scattering transform network is used for fingerprint recognition based on wavelet transform in [3]. The scattering transform compute local image descriptors with three operations like Wavelet decompositions, complex modulus and local averaging, SVM classifier is used for template matching [3]. In [4], the Global information fusion scheme is used for finger print recognition. DOST is used to extract the instantaneous phase difference. The basic textures like magnitude, phase and orientation are extracted by using SDOST.2D Gabor filters and dual terry complex wavelet transform are used for the extraction of image local orientation information. The DWT is better for the compression of images. DTCWT improves the directionality and reduces the shift sensitivity [5].

The 2D gabor wavelet transform performs multi resolution and multi orientation time frequency analysis [6]. Global thresholding is used for the segmentation of blood vessels. It provides high sensitivity and specificity values and better diagnosis [6].An automatic retina verification framework is proposed for biometric identification [7]. The top hat and bottom hat filtering is used to enhance the blood vessel and filter the background noise. Thresholding is used for the separation of light and dark region. The Fourier transform of the image is obtained by gabor filter [8]. The retinal authentication scheme is used to identify the blood vessels on the thin nerves. It gives $100 \%, 96.3 \%$ and $91 \%$ of recognition rates for DRIVE, VARIA and STARE data sets respectively [9][10]. The graph cut segmentation technique is used to detect the layers of the retinal images and the bias correction algorithm is used to reduce the noise [11]. The morphological open function is used to eliminate background variations [12].

The unimodal biometric recognition system has its own drawbacks such as feature distinctiveness, improper feature acquisition, processing error etc. Hence a single biometric trait is not enough for proper authentication. In biometric fusion, the score level, rank level and decision level fusion need series of processing that leads more time complexity and also it requires more space for storing all the input images that leads to space complexity. In order to overcome the limitations there is a need for feature level fusion for perfect authentication with less time and space complexity. 


\section{MATERIALS AND METHODS}

\section{PROPOSED SYSTEM}

The block diagram of the proposed authentication system is given below

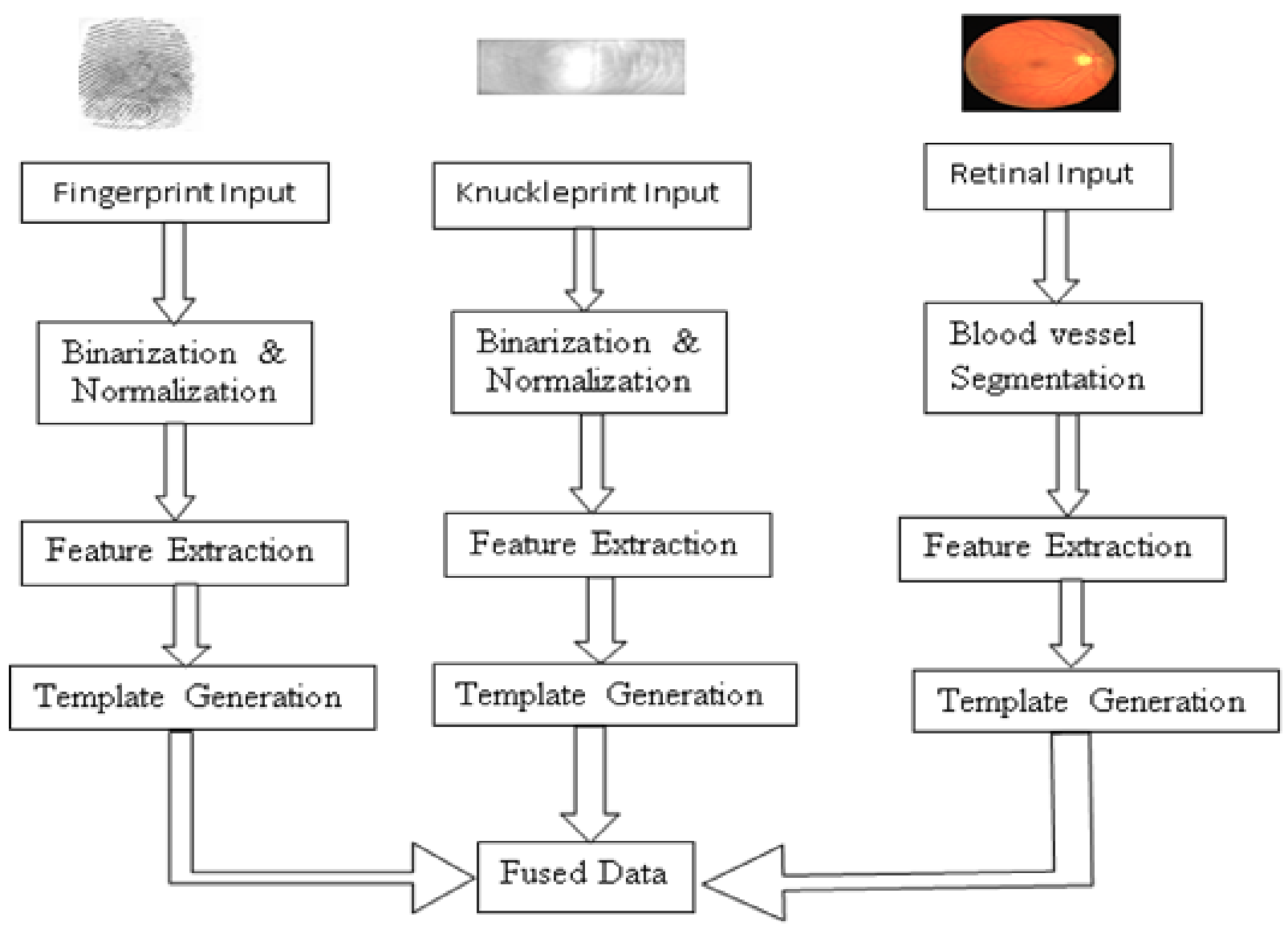

Figure: i) The block diagram of the proposed authentication system.

\section{Feature Extraction}

\section{i)Fingerprint}

In this work the level II features like singular points (core and delta point), bifurcation and the ridge ending are considered.

a) Fingerprint acquisition by means of sensor

b) Preprocessing is done for image enhancement. In this,

$\rightarrow$ Grayscale image is binarized and then

$\rightarrow$ The binarized image is normalized

$\rightarrow$ Orientation of the fingerprint is obtained from the normalized image

c) Reconstruction of the image is done by the orientation.

d) Ridge filter enhancement is used to eradicate the spurious minutiae present on the edges of the finger print.

e) Morphological operation and structural elements are used for thinning.

f) Features are extracted.

The singular points are identified using the equlidien distance method. The distance between the core and the minutiae points are obtained. In order to calculate the angle between the core and minutiae the slope of core and minutiae, and the slope of core and delta are considered. The extracted features along with their angle, distance and type of minutiae are arranged in the form of a template. 
The knuckle print is the outer surface of the finger. It has got lot of creases and wrinkles. The feature extraction steps of knuckle print is almost similar to the feature extraction of the finger print. The difference is that, the knuckle print is considered in horizontal direction and a window is drawn considering the outer edges of the knuckle. The window is divided into four equal parts and the midpoint of the window is considered as the midpoint of the knuckle. The figure: ii) (A) shows the input image of knuckle and figure, ii) (B) shows the ROI in the input image.

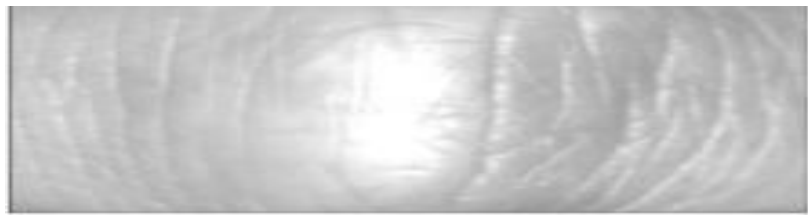

A

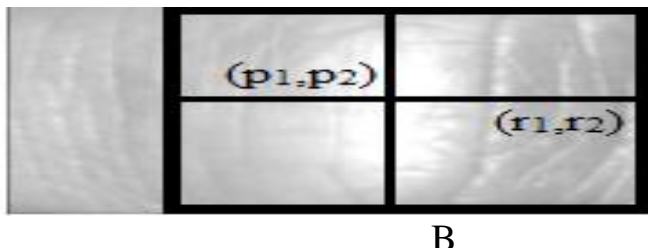

B

Figure: ii) (A) Input of knuckle print (B) ROI

The feature extraction of the knuckle print is done by calculating the midpoint and the reference point. The distance between the midpoint and the minutiae point are calculated. In order to obtain the angle, the slope is calculated between the midpoint of the knuckle and the minutiae point. The slope is calculated between the midpoint of the knuckle and the reference point. The extracted features along with their angle, distance and type of minutiae are arranged in the form of a template.

iii) Retina

The fundus retinal database is used for the analysis. The images in the database are the colour images, so the three channels red, green and blue are separated for better segmentation results. The green channel has better visibility of the blood vessels, hence, the green channel is considered for further processing.

a) De-noising: Top hat filter and bottom hat filters are used

b) Enhancement: Adaptive Histogram Equalization is used.

c) Segmentation: Otsu thresholding with morphological operator.

The adaptive histogram equalization is done to increase the contrast of the image. The Otsu thresholding with morphological operator is used for the accurate segmentation of the blood vessels. The segmented image is then normalized and the minutiae extraction is done. In this, the point of bifurcation of the blood vessels and the blood vessel endings are considered as features. The optical disk is considered as the centre point and the Euclidian distance is calculated between the optic disk and the minutiae. The main nerve is identified depending on its thickness and its ending point is considered as the reference point, for determining the angle of the minutiae points. The extracted features along with their angle, distance and type of minutiae are arranged in the form of a template.

\section{Feature level Fusion}

The fused template set which are obtained from the fingerprint, knuckle print and the retina along with their angle, distance and type are fused by means of two dimensional arrays to form a unique template and this unique fused template is stored in the data base for further authentication process.

$\mathrm{C}_{\mathrm{ij}}=\mathrm{a}_{\mathrm{ij}} * \mathrm{~b}_{\mathrm{ij}}$

Where, $\mathrm{a}_{\mathrm{ij}}$ is the array of fingerprint and knuckle print template.

$b_{i j}$ is the array of retinal template.

$\mathrm{C}=\mathrm{a}_{\mathrm{ik}}{ }^{*} \mathrm{~b}_{\mathrm{kj}}+\mathrm{c}_{\mathrm{ij}}$ 


\section{RESULTS AND DISCUSSIONS}

\section{i)Fingerprint}

The FVC database is the publicity available largest database for fingerprint. This dataset contains of 3843 images and the thumb and middle finger are considered. The images of each finger are captured in 8 different instances. The FVC 2000 to FVC 2004 database was used for the evaluation of the proposed authentication system. The fingerprints were categorized as good, medium and bad depending upon the visual perception. The features were extracted accurately and the false minutiae were eradicated. The obtained outputs are as follows:

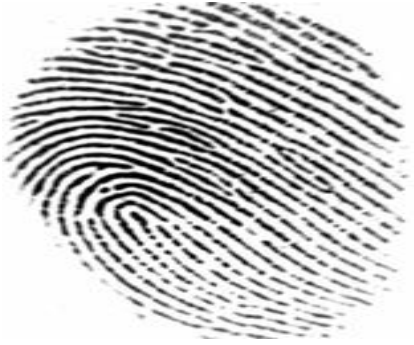

A

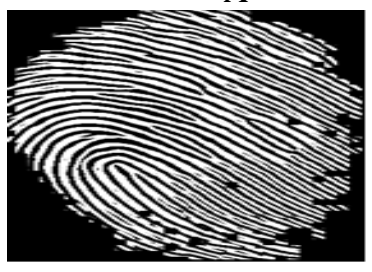

$\mathrm{D}$

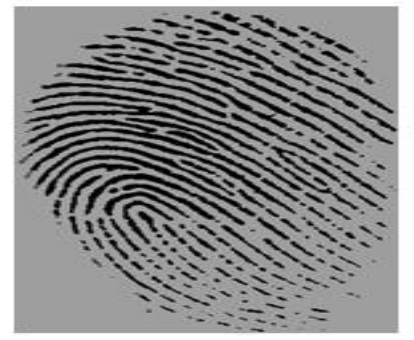

B

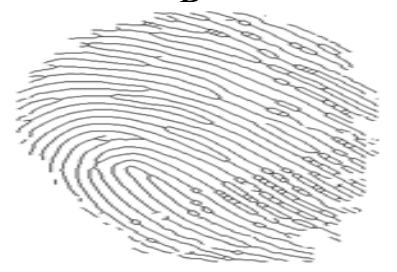

E

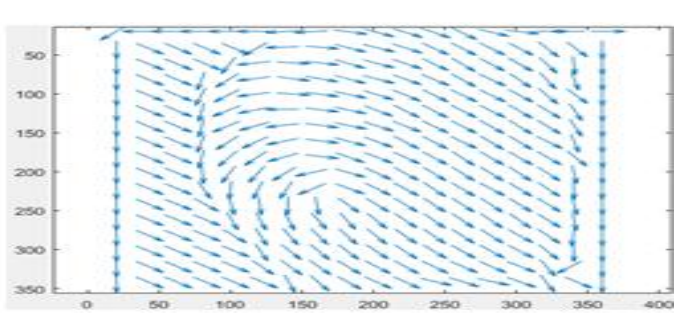

$\mathrm{C}$

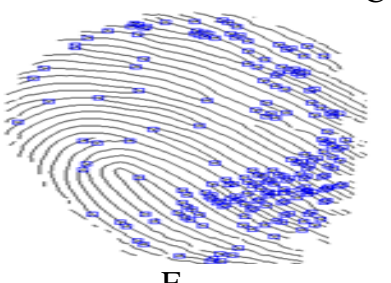

$\mathrm{F}$

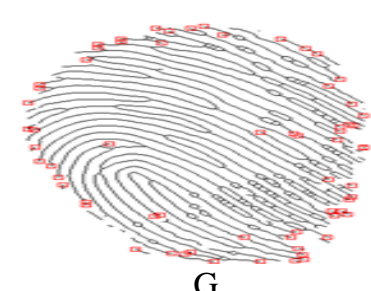

G

Figure: iii) A, B, C, D, E are the different stages of fingerprint preprocessing and F, G are the extracted features

ii)

Knuckle Print

The Hong Kong polytechnic university database was used for the evaluation of the proposed authentication system. This is the largest publicity available database and the images are obtained from 165 subjects, the images of the index finger and the middle finger of the both hands are acquired. The images are acquired at 12 different instances for each finger. The results of the feature extraction are as follows:

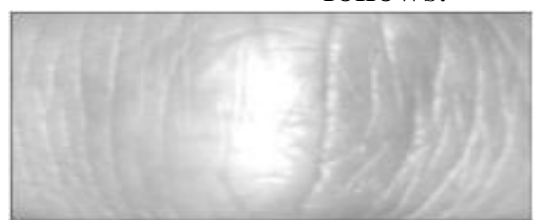

A

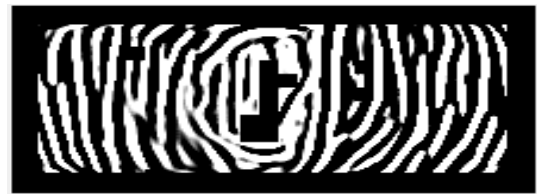

$\mathrm{D}$

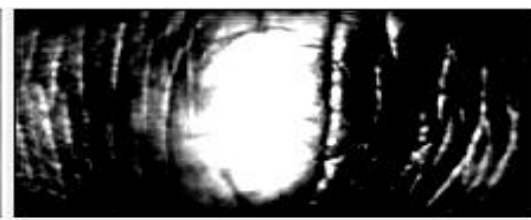

$\mathrm{B}$

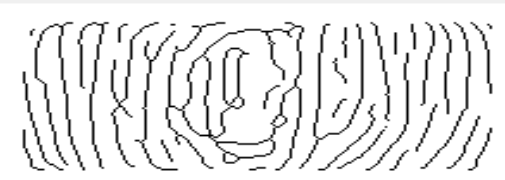

$\mathrm{E}$
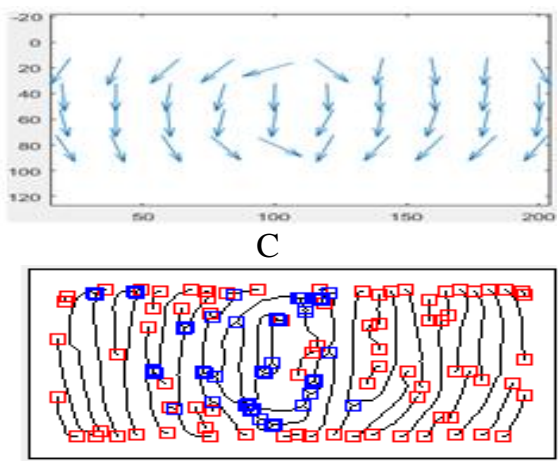

$\mathrm{F}$

Figure: iv) A, B, C, D, E are the different preprocessing stages and F is the feature extracted from the knuckle print

\section{iii) Retina}

The fundus and the drive database are used for the evaluation of the proposed system. Both the database together contains 56 images of the retina which are acquired using the fundus camera. 


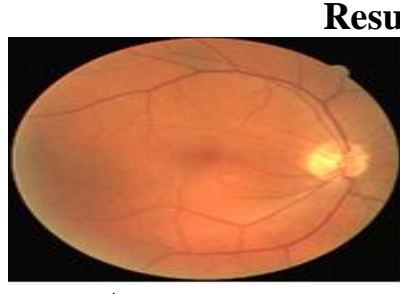

A

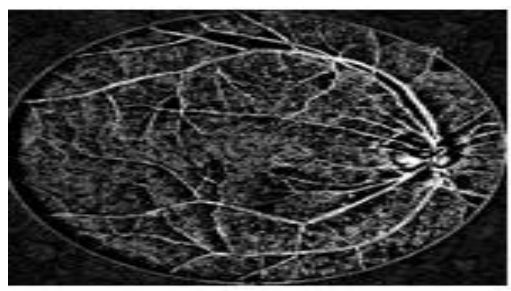

E

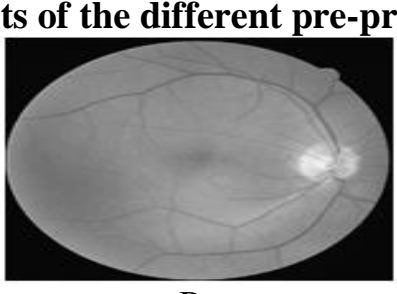

B

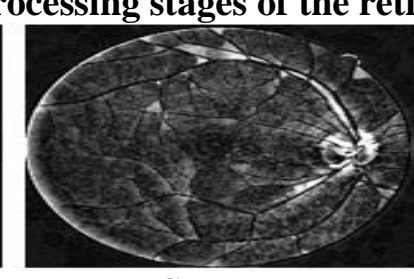

C

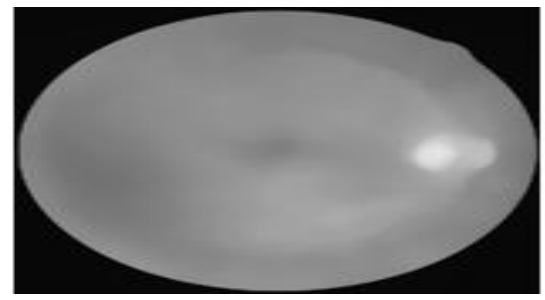

$\mathrm{F}$

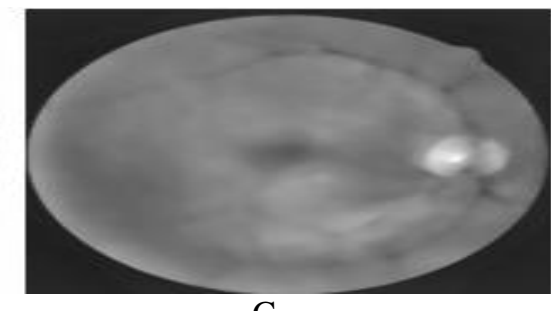

G

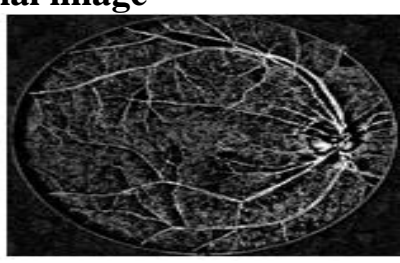

D

Figure: v) A) Input Retina Image, B) Gray image of Retina, C) Contrast adjusted top hat transform, D) Contrast adjusted bottom hat transform, E) Contrast adjusted image, F) After applying median filter ,G) After applying CLAHE.

Segmentation of the blood vessel and the feature extraction of the retinal image

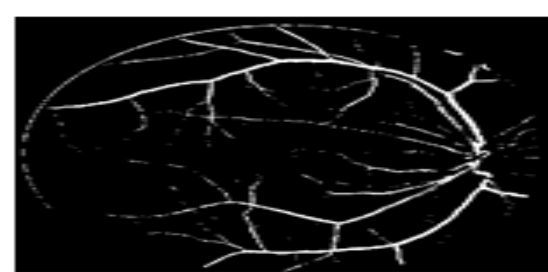

$\mathrm{H}$

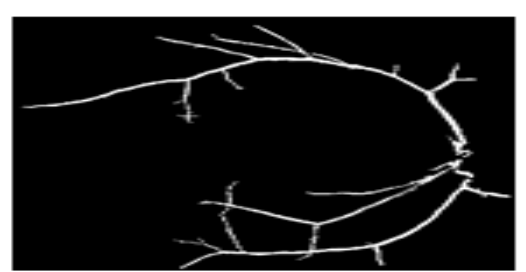

I

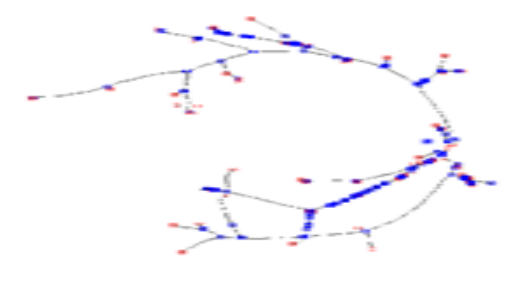

$\mathrm{J}$

Figure: vi) H) Segmentation of blood vessels, I) Extraction of the largest blob image, J) Features extracted with optic disk as centre point

Template obtained by fusing the fused data set of the fingerprint and the knuckle print with the data of the retina.

Table: i) Fused data set of the Fingerprint, the Knuckle print and the Retina

\begin{tabular}{|l|l|l|l|}
\hline 1164.491 & 2116.418 & 105.981 & 60.422 \\
\hline 1682.09 & 1150.111 & 180.050 & 56.789 \\
\hline 1857.80 & 1120.131 & 110.574 & 59.881 \\
\hline 1780.010 & 1224.001 & 179.568 & 40.422 \\
\hline 1981.141 & 1471.804 & 150.410 & 45.398 \\
\hline 1587.181 & 1001.000 & 154.358 & 70.891 \\
\hline 1684.116 & 138.987 & 147.695 & 65.782 \\
\hline
\end{tabular}

A unique fused data set is generated for all the enrolled identity and during the authentication process the query image is obtained and the extracted features are matched with the template present in the data base. If the similarity is above $98 \%$ then the person is more authenticated. The algorithm is checked with more samples and the result shows that the efficiency is high. Thus, the space and the time complexity is reduced. 
Feature Level for Personal Authentication

\section{CONCLUSION}

In the proposed system, the unique features obtained from the fingerprint, the knuckle print and the retina are fused using two dimensional array based fusion method. This shows, the final template obtained are more secure and unique. The result shows that it is more unique than the unimodal and other biometric fusion methods. The GAR is $95.9 \%$ for the feature fusion utilizing Gabor filter over Finger print, Knuckle print and retina.

\section{REFERENCES}

[1] Muthukumar Arunachalam and Kannan Subramanian "AES Based Multimodal Biometric Authentication using Cryptographic Level Fusion with Fingerprint and Finger Knuckle Print" The International Arab Journal of Information Technology, Vol. 12, No. 5, September 2015

[2] Divyakant T. Meva and C. K. Kumbharana "Comparative Study of Different Fusion Techniques in Multimodal Biometric Authentication” International Journal of Computer Applications (0975 - 8887) Volume 66-No.19, March 2013

[3]Shervin Minaee and Yao Wang

"FingerprintRecognitionUsingTranslationInvariantScatteringNetwork" arXiv:1509.03542v3 [cs.CV] 26 Nov 2015

[4] Mahesh Kumar, N.B. and K. Premalatha "Finger Knuckle-Print Identification Based on Local and Global Feature Extraction Using SDOST"American Journal of Applied Sciences 11 (6): 929-938, 2014

[5] Hanna Paulose and Harbinder Singh "Finger Knuckle Analysis: Gabor Vs DTCWT" International Journal on Recent and Innovation Trends in Computing and Communication volume:3 Issue :5 May 2015

[6] Prof.S.V.Pattalwar, Mr.L.SKalkonde "A Review on Detection of Extra Blood Vessel Growth in Retinal Images using Wavelet Based 2D Gabor Contourlet Transform" International Journal of Science, Engineering and Technology Research(IJSETR),volume 3,Issue4,April2014

[7] Lincy S.L Monisha and Dr.C.Seldev Christopher Professor, "Biometric Identification Using Retina Scan” International Journal of Advanced Research Trends in Engineering and Technology (IJARTET) Vol. II, Special Issue XXIII, March 2015

[8] Jose Ignacio Orlando and Maraiana del fresno "Reviewing pre-processing and feature Extraction Techniques for Retinal Blood Vessels segmentation in Fundus Image" Computer vision and Image processing 2015

[9] L. Latha, M. Pabitha and S. Thangasamy "Effectual Human Authentication for Critical Security Applications Using Retinal Images" ICTACT Journal on Image and Video Processing, November 2010, Issue: 02

[10]YitianZhao, YonghuaiLiu,XiangqianWu,SimonP.Harding, YalinZheng "RetinalVesselSeg mentation:AnEfficientGraphCutApproachwithRetinexandLocal Phase" PLOSONE April1,2015

[11] D.Kaba, Y.Wang, C.Wang, X.Liu, H.Zhu, A.G. Salazar-Gonzalez,andY.Li1 "Retinalayersegmentationusingkernel graphcutsandcontinuousmax-flow" Optical Society of America Vol. 23, No. 6, 23 March 2015

[12] Divya A Sajjan "Analyzing the Retinal Blood Vessels Extraction and Bifurcation Points in Color Retina Fundus Image” International Journal of Science and Research (IJSR) June 2014

Received: February 03, 2016; Accepted: July 14, 2016 